\title{
Surgical management of childhood bronchiectasis due to infectious disease
}

Gokhan Haciibrahimoglu, MD

Mithat Fazlioglu, MD

Aysun Olcmen, MD

Atilla Gurses, MD

Mehmet Ali Bedirhan, MD

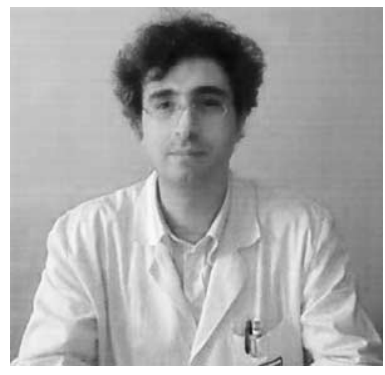

Dr Haciibrahimoglu
Background: The purpose of this study was to estimate operative risk and to identify indicators of adverse prognosis in patients undergoing resection for childhood bronchiectasis.

Methods: From January 1985 to February 2001, patients undergoing resection for bronchiectasis were studied. The indications for operation were failure of medical therapy in 33 patients $(94.2 \%)$ and hemoptysis in $2(5.7 \%)$. The mean duration of symptoms was 4.2 years (range, 1-9 years). Surgical treatment included lobectomy in 17 patients $(48.5 \%)$, pneumonectomy in $7(20 \%)$, lobectomy plus segmentectomy in $5(14.2 \%)$, bilobectomy in $2(5.7 \%)$, and segmentectomy in $4(11.4 \%)$.

Results: The operative mortality rate was $2.8 \%$, and the morbidity rate was $17.6 \%$. The mean follow-up in 34 patients was 5.4 years (range, 1-12 years). Overall, 22 patients $(64.7 \%)$ were asymptomatic after surgery. Clinical improvement was noticed in 8 patients (23.5\%), and no improvement was noticed in 4 (11.7\%). Complete resection resulted in a significantly better clinical outcome than incomplete resection $(P<.05)$.

Conclusions: Surgery for childhood bronchiectasis can be performed with low mortality and morbidity. Complete resection should be performed when possible.

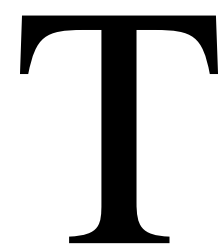

he incidence of bronchiectasis, which is defined as irreversible dilatation of the bronchial tree, has decreased considerably since the advent of antibiotic therapy. The disease not only affects the structure of the bronchial tree, but also alters pulmonary hemodynamics and causes nonperfused lung fields. Thus, surgical resection is required in some patients despite maximum medical support to control the symptoms and progression of the disease, and it is still an indication for lung resection before puberty. Bronchiectasis occurs for a number of congenital and acquired reasons, and it is predominantly a pediatric disease. ${ }^{1,2}$

In postinfectious bronchiectasis, in most cases one can elicit a history of pneumonia developing as a complication of measles, whooping cough, or another contagious disease of childhood; it is thought that the bacterial pneumonia and associated atelectasis are responsible for destruction and dilatation of the bronchial walls. We think that postinfectious bronchiectasis is a different entity. In spite of widely known etiologic factors, the causes could not be determined in $29 \%$ to $45 \%$ of cases. ${ }^{3}$ In our country, pneumonia (30\%) and tuberculosis (11\%) are the most common causes. ${ }^{4}$ The purpose of this study was to evaluate the indications, postoperative complications, and survival with lung resections performed in children because of postinfectious bronchiectasis. 
TABLE 1. Symptoms

\begin{tabular}{lc}
\hline Symptom & $\mathbf{n ~ ( \% )}$ \\
\hline Recurrence of pulmonary infection & $33(94.2 \%)$ \\
Productive cough & $32(91.4 \%)$ \\
Fetid sputum & $28(80 \%)$ \\
Hemoptysis & $4(11.4 \%)$
\end{tabular}

\section{Patients and Methods}

The patient notes of 35 patients who underwent lung resection at our institute from 1985 to 2001 were retrospectively reviewed. The records were analyzed for age, sex, symptoms, type of resection, operative morbidity and mortality, and long-term outcome. Patients older than 14 years of age were excluded. Only patients who had bronchopulmonary infections as an etiologic factor were included in this review.

Patients who presented to chest disease clinics with pulmonary infections and excessive amounts of sputum production underwent further evaluation, including pulmonary artery and lateral radiographs and computed tomographic (CT) scan. Preoperative pulmonary function studies were affected by a lack of cooperation with younger patients (5-13 years) and could not be performed completely. Bronchography was used at the beginning of the series. The diagnosis was made on CT scan in all patients. The CT criteria for diagnosing bronchiectasis included a bronchial diameter greater than that of the accompanying pulmonary artery, lack of tapering of the bronchial lumen, and visualization of bronchi in the periphery of the lungs. Ventilation/perfusion ( $\dot{\mathrm{V} / \mathrm{Q}})$ scintigraphy was performed at the end of the series. The congenital origins in childhood bronchiectasis were investigated. Before surgery, a sweat test was performed for cystic fibrosis. Paranasal sinusitis was investigated.

Preoperative medical treatment, which included appropriate antibiotic therapy and postural drainage, was supervised by chest physicians. Sputum was cultured for bacterial examination before surgery.

Surgical treatment was considered if the medical treatment failed. We considered that the treatment was a failure if sputum production persisted despite several ( 2 or 3 ) courses of treatment and if there was a limited extent of disease on CT. As a rule, the extent of diseased lung to justify operation must be localized to achieve complete resection. The decision for the volume of the resected lung was based on radiologic findings rather than on thoracotomy. Detailed investigation was undertaken of the middle lobe in patients having right thoracotomy and of lingular segments in patients having left thoracotomy.

All specimens had pathologic confirmation of bronchiectasis. The pulmonary resection was considered complete if the patient was believed to be free of bronchiectasis after thoracotomy. After surgery, patients were observed by a team that included chest physicians and surgeons. They were examined 4 times a year for 3 years and twice thereafter. High-resolution CT (HRCT) examinations were performed once a year. Immunization, including pneumococcus and influenza vaccines, was performed routinely. Clinical improvement can be defined as a decrease in symptoms of infection episodes when compared with the number of episodes preoperatively. This means that symptoms were observed only one time a year, which can be controlled with antibiotics.
TABLE 2. Bacteriology

\begin{tabular}{lc}
\hline Germ & $\mathbf{n ~ ( \% )}$ \\
\hline Staphylococcus aureus & $17(48.5 \%)$ \\
Pseudomonas aeruginosa & $6(17.1 \%)$ \\
Klebsiella pneumoniae & $3(8.5 \%)$ \\
Streptococcus pneumoniae & $2(5.7 \%)$ \\
\hline
\end{tabular}

\section{Operative Technique}

Rigid bronchoscopy was performed in all patients. We used bronchial blockers for isolation of the airway to prevent spillage of secretions when performing the operation for bronchiectasis in children. All procedures were through a classic posterolateral thoracotomy. Lung resection was performed. Bronchial closure was preferably achieved with manual interrupted semiabsorbable sutures, and the bronchial stump was covered with adjacent tissues in some patients, depending on the surgeons' preference.

\section{Findings}

The mean age of the patients at the time of thoracotomy was 10.6 years (range, 5-13 years). There were 18 male and 17 female patients. The most common symptoms were recurrent infections; the average duration of symptoms was 4.2 years (range, 1-9 years; Table 1).

In physical examination of these children, according to growth percentiles, musculoskeletal development retardation was noted. These children were the ones who were under the 3rd percentile of the growth percentile curve. Posteroanterior and lateral chest radiographs were first made as preoperative diagnostic studies in all patients. Contrast bronchography was performed in 8 patients $(22.8 \%)$, and HRCT was performed in 27 patients $(77.1 \%)$. Bronchography was used mostly in the beginning of the series. $\dot{V} / \mathbf{Q}$ scintigraphy was performed in 10 patients $(28 \%)$. On $\dot{\mathrm{V}} / \mathrm{Q}$ scintigraphy, perfusion and ventilation were both impaired, but ventilation was more affected. Preoperative bronchoscopy was performed routinely in all patients. The cause of secondary bronchiectasis in 5 of the patients was central obstruction or stenosis. We had 5 patients with paranasal sinusitis. These patients had nasal polyps due to chronic sinusitis. Before the operation, these cases were evaluated by a specialist, and 3 of these 5 patients underwent operation. Afterward, they were operated on for bronchiectasis. During their long follow-ups, they had no problems, whereas the other 2 cases had occasional infections.

Bacteria were preoperatively proven in 28 patients $(80 \%)$. These are shown in Table 2. An antibiotic was given to all patients according to the culture antibiogram. A second-generation cephalosporin was administered to patients before surgery (ie, empiric antibiotic treatment).

The disease was bilateral in 2 patients $(5.7 \%)$ and unilateral in $33(94.2 \%)$ : right chest in $12(36.3 \%)$ and left chest in $21(63.6 \%)$. Bronchiectasis involvement was predominantly in the basilar segments in 21 patients $(60 \%)$, lingular segments in $9(25.7 \%)$, and upper lobe segments in 7 (20\%).

The indication for pulmonary resection was made in 33 patients (94.2\%) because of failure in medical therapy and in $2(5.7 \%)$, of 
TABLE 3. Symptomatic status of 34 patients

\begin{tabular}{lclr}
\hline Symptoms & $\begin{array}{c}\text { Complete } \\
\text { resection }\end{array}$ & $\begin{array}{c}\text { Incomplete } \\
\text { resection }\end{array}$ & \multicolumn{1}{c}{ Total } \\
\hline Asymptomatic & $21(75 \%)$ & $1(16.6 \%)$ & $22(64.7 \%)$ \\
Clinical improvement & $6(21.4 \%)$ & $2(33.3 \%)$ & $8(23.5 \%)$ \\
No improvement & $1(3.5 \%)$ & $3(50 \%)$ & $4(11.7 \%)$ \\
Total & $28(100 \%)$ & $6(100 \%)$ & $34(100 \%)$ \\
\hline
\end{tabular}

chronic hemoptysis. Seventeen patients $(48.5 \%)$ underwent lobectomy, and $7(20 \%)$ underwent pneumonectomy. In 5 patients (14.2\%) lobectomy plus segmentectomy was performed, in 4 patients $(11.4 \%)$ segmentectomy was performed, and in 2 others $(5.7 \%)$ bilobectomy was performed. We performed bilateral resection in 2 patients. The lower lobectomies were performed in 2 stages. In the morphologic classification, 24 cases $(68.5 \%)$ were cylindrical, 8 cases $(22.8 \%)$ were saccular, and 3 cases $(8.7 \%)$ were varicose bronchiectasis.

\section{Statistical Analysis}

Functional results were compared by using a $\chi^{2}$ test.

\section{Results}

One patient (2.8\%) died the second day after surgery. Right lower bilobectomy had been performed in this patient. The cause of death was unexpected sudden arrhythmia and cardiac arrest.

Complications occurred in 6 patients (17.6\%). These complications included atelectasis in 3 patients $(8.8 \%)$, empyema in $2(5.8 \%)$, and prolonged air leak ( $>7$ days ) in 1 (2.9\%). The follow-up was complete for 34 patients (100\%) - 28 who had complete resection $(82.3 \%)$ and 6 who had incomplete resection (17.7\%)—with a mean time of 5.4 years (range, 1-12 years).

We found that there was progressive overinflation of the remaining lung during the first year after pneumonectomy. The overinflation remained stable thereafter. Anterior herniation of the remaining lung with mediastinal shift was seen in long-term follow-up. Anterior herniation crossed the midline anterior to the heart. Cardiac functions of the patients showed no pathologic evidence. After surgery for the patients who underwent pneumonectomy, we observed that the hemithorax at that side became smaller and that slight scoliosis occurred. The growth pattern accelerated but has not yet attained normal percentiles in these patients.

The patients underwent direct evaluation. Twenty-two (64.7\%) were asymptomatic, and 8 (23.5\%) had improved compared with their preoperative symptoms. Only 4 patients (11.7\%) showed no improvement (Table 3). The best clinical improvement of this group was observed in patients with complete resection of the disease (27 of 28 patients); only 1 patient did not improve. In contrast, only 1 of 6 patients who had incomplete resection was asymptomatic, and 3 patients (50\%) showed no improvement. A case of

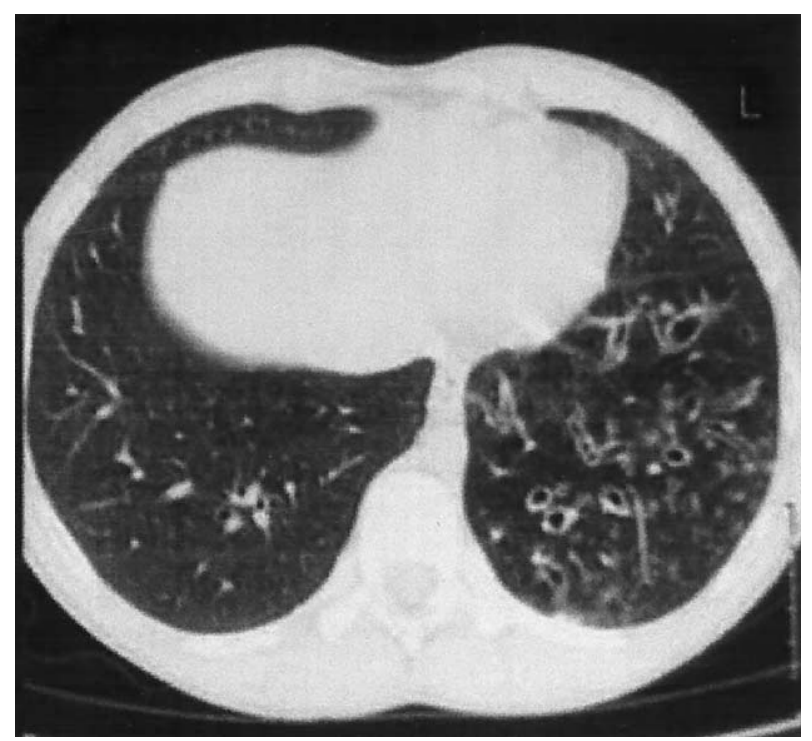

Figure 1. Preoperative HRCT of the patient in whom left lobectomy was performed.

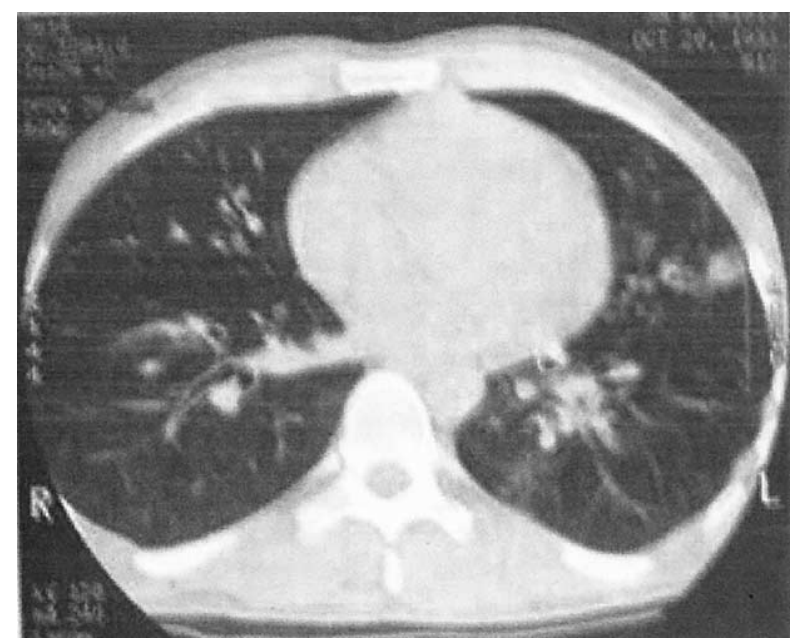

Figure 2. The right middle lobe and lingula show bronchiectatic areas after left lower lobectomy (same patient as in Figure 1; incomplete resection).

incomplete resection with HRCT taken before surgery and during the first year after surgery is shown in Figures 1 and 2. Complete resection gave a significantly better result than incomplete resection $(P<.05)$. During follow-up of 6 patients who underwent incomplete resection, we observed nonlocalized bronchiectatic areas.

In the follow-up, we performed V/Q scans in 10 patients after 1 year. Improvement was especially noted in ventilation. Ventilation scans made before surgery and the first year after surgery, illustrating this situation, are shown in Figures 3 and 4. 


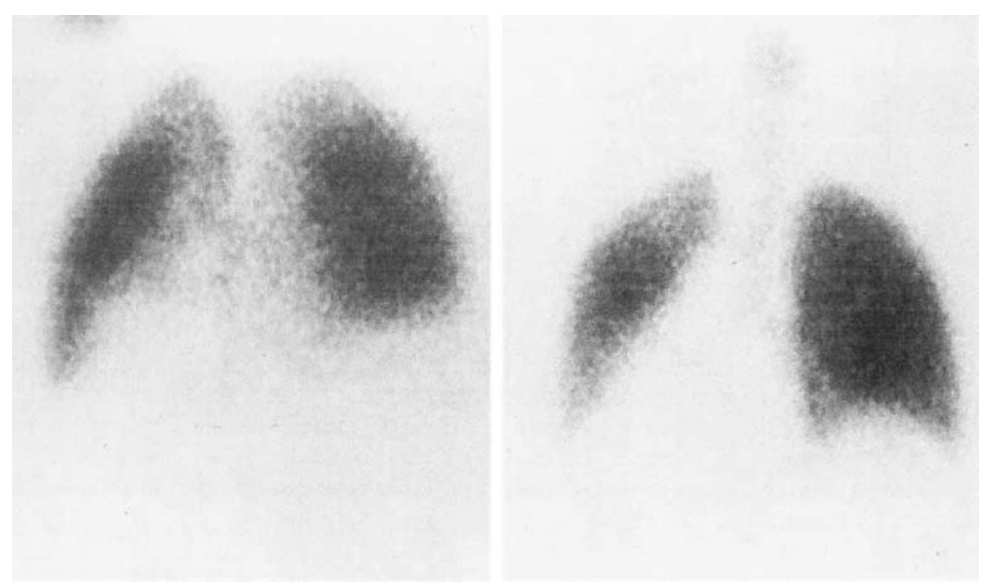

Figure 3. There is a complete absence of ventilation of the left lower lobe in $\mathbf{V} / \hat{Q}$ scan. Left, Ventilation scan. Right, Perfusion scan.

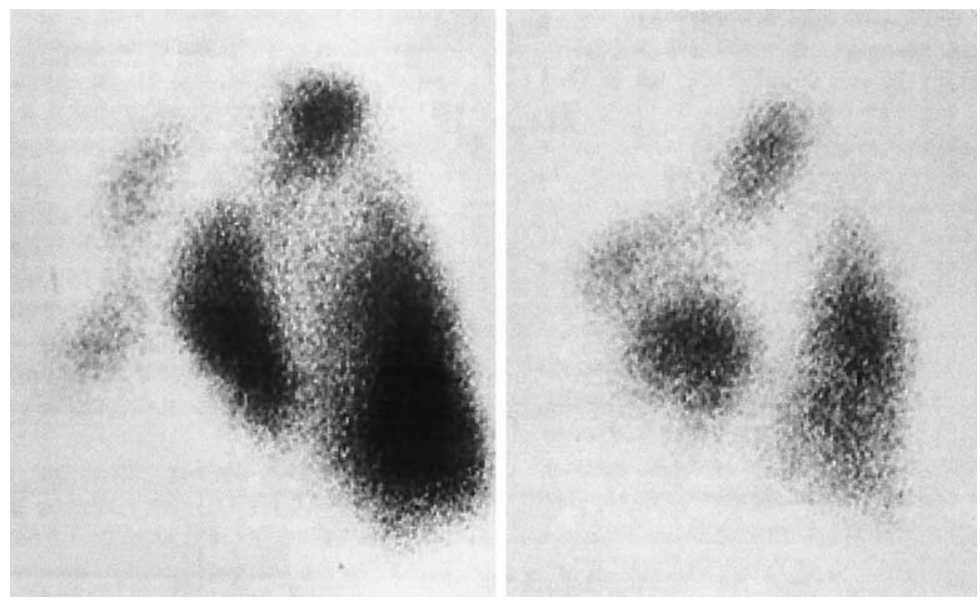

Figure 4. After left lobectomy, the ventilation scan was improved.

\section{Discussion}

Bronchiectasis is a chronic necrotizing condition of the bronchi and bronchioles that leads to irreversible abnormal dilatation of the airways. The morphologic classes of bronchiectasis are cylindrical, varicose, and saccular (cystic). It covers a spectrum from mild (cylindrical) to the most severe form of the disease (cystic). Ashour ${ }^{5}$ stated that pulmonary perfusion was retained in the area of cylindrical changes and that, therefore, this type was not a primary indication for surgical management. Two thirds of the patients in our series had cylindrical bronchiectasis and nevertheless had symptoms that were resistant to medical therapy; these were alleviated successfully by surgical management. Furthermore, patients with a cylindrical type acquired a better prognosis after operation than those who had other types. Thus, we recommend surgical resection, even though the diseased lung has cylindrical change. Our experience sug- gests that patient symptoms indicate surgical resection rather than radiologic findings.

In this study, we observed that the basal segments of the lower lobes, especially the posterobasal segments, were the most frequently affected (60\%). We had expected this to occur more frequently on the left side, because the retaining of secretions can be thought to more easily occur in the left side (because of its smaller diameter and greater angle and its spatial relationship with the left pulmonary artery). ${ }^{6,7}$

Bronchiectasis is usually caused by bronchopulmonary infection or bronchial obstruction; approximately half of patients can trace the onset of symptoms to a childhood infection. Primarily the symptoms are chronic cough and fetid sputum. In our series, Staphylococcus aureus and Pseudomonas aeruginosa were the most common organisms isolated from sputum.

The chest radiograph is a poorly sensitive diagnostic instrument, more than $20 \%$ of the limited form of which is 
regarded as normal, and we have used it routinely as a screening tool. Especially when the chest radiograph shows circular or honeycombing appearance, there is a high suspicion of bronchiectasis. The classification of bronchiectasis is less important than determining the extent of disease. The surgeon must identify the segment that is affected, because modern surgical techniques frequently permit preservation of 1 or more healthy pulmonary segments of a lobe in which bronchiectasis is present. Classically, bronchography has been routinely used for confirming the diagnosis and defining the extent of disease. The use of bronchography has been steadily decreasing (it was used in 8 [22.8\%] of our patients, mostly in the beginning of the series), and it has been progressively replaced by HRCT. The lack of distal tapering of the bronchial lumen has been considered to be the most sensitive criterion for indicating bronchiectasis. ${ }^{8}$

Preoperative bronchoscopy should be routinely performed to rule out foreign body, neoplasm, or stricture. We operated on 5 patients with secondary bronchiectasis caused by central obstruction or stenosis. Preoperative acute suppurative bronchitis was treated aggressively with appropriate antibiotics and postural drainage. A bronchial blocker was used to avoid contralateral contamination of secretions. The bronchial stump was manually sutured by using semiabsorbable materials in all patients, and bronchial reinforcement was performed in some patients, depending on the surgeons' preference.

The role of pulmonary resection has evolved from early curative resection for all patients ${ }^{9}$ to a more palliative approach limited to either those patients with medically resistant disease or those with complications. The occurrence of complications is $9.4 \%$ to $24.6 \%$ in the current literature ${ }^{9-11}$; this range is comparable to our rate $(17.6 \%)$. In our series, complications were seen in 6 patients, and all were treated by a conservative approach. Atelectasis depended on sputum retention. Sputum retention is common because patients with this disease can have problems with ciliary motion and postoperative expectoration, which would easily be disrupted.

As others ${ }^{12}$ have demonstrated, bilateral bronchiectasis is not a contraindication to operation, and even incomplete resection often results in considerable alleviation. In our series, we performed bilateral resection in only 2 patients, for whom the lower lobectomies were performed in 2 stages.
The aims of surgical therapy for bronchiectasis are to improve the quality of life in patients who have debilitating pulmonary symptoms despite medical treatment and to prevent complications such as empyema, recurrent hemoptysis, and lung abscess. Patients with clearly symptomatic, welllocalized childhood bronchiectasis are ideal surgical candidates. ${ }^{11,13,14}$ Resection can be performed with almost no risks and a high expectation for cure.

In conclusion, surgical treatment for childhood bronchiectasis should be limited to patients with well-localized disease. It can be performed with a definite strategy and strict criteria. Complete resection should be performed whenever possible. Preservation of as much lung parenchyma as possible is important. The basic guidelines for operative therapy of bronchiectasis are infection control, preoperative bronchoscopy, precise anatomic diagnosis, and precise anatomic resection.

\section{References}

1. Paré JAP, Fraser RG. Synopsis of diseases of the chest. 2nd ed. Philadelphia: Saunders; 1994. p. 677-89.

2. Swartz MN. Bronchiectasis. In: Fishman AP, editor. Pulmonary diseases and disorders. New York: McGraw-Hill; 1988. p. 1553-81.

3. Gracia JD, Rodrigor MJ, Morelle F, et al. Ig G subclass deficiencies associated with bronchiectasis. Am J Respir Crit Care Med. 1996;153: 650-5.

4. Ilvan A, Bozkanat E, Kartaloglu Z, et al. Son 5 yil icinde bronkografi ile tani konulmus bronsektazi olgularinin degerlendirilmesi. Solunum. 1995;19:842-7.

5. Ashour M. Hemodynamic alterations in bronchiectasis: a base for a new classification of the disease. J Thorac Cardiovasc Surg. 1996; 112:328-34.

6. Ashour M, Pandya I. Unilateral post-tuberculosis lung destruction: the left bronchus syndrome. Thorax. 1990;45:210-2.

7. Ashour M. The anatomy of the bronchus syndrome. Clin Anat. 1995; 8:256-61.

8. Lucidorme O, Grenier P, Coche E, Lenoir S, Aubert B, Beigelman C. Bronchiectasis: comparative assessment with thin-section CT and helical CT. Radiology. 1996;200:673-9.

9. Ripe E. Bronchiectasis: a follow up study after surgical treatment. Scand J Respir Dis. 1971;52:96-101.

10. Annest LS, Kratz JM, Crawford FA. Current results of treatment of bronchiectasis. J Thorac Cardiovasc Surg. 1992;83:546-50.

11. Wilson JF, Decker AM. Surgical management of childhood bronchiectasis. Ann Surg. 1982;195:354-63.

12. Fujimoto T, Hillejan L, Stamatis G. Current strategy for surgical management of bronchiectasis. Ann Thorac Surg. 2001;72:1711-5.

13. Agasthian T, Deschamps C, Trastek VF, Allen MS, Pairolero PC. Surgical management of bronchiectasis. Ann Thorac Surg. 1996;62: 976-80.

14. Prieto D, Bernardo J, Matos MJ, Eugenio L, Antunes M. Surgery for bronchiectasis. Eur J Cardiothorac Surg. 2001;20:19-24. 